\title{
HOW THE INTERVAL BETWEEN PRIMARY AND BOOSTER VACCINATION AFFECTS LONG-TERM DISEASE DYNAMICS*
}

\author{
AFFAN SHOUKAT \\ Agent-Based Modelling Laboratory \\ York University, Toronto \\ Ontario M3J 1P3, Canada \\ E-mail: affans@yorku.ca \\ AQUINO L. ESPINDOLA \\ Departamento de Física \\ Instituto de Ciências Exatas - ICEx \\ Universidade Federal Fluminense \\ Volta Redonda, RJ, Brazil, 27.213-145 \\ E-mail: aquinoespindola@id.uff.br \\ GERGELY RÖST \\ Bolyai Institute, University of Szeged \\ 6720 Szeged, Hungary \\ E-mail: rost@math.u-szeged.hu \\ SEYED M. MOGHADAS \\ Agent-Based Modelling Laboratory \\ York University, Toronto \\ Ontario M3J 1P3, Canada \\ E-mail: moghadas@yorku.ca
}

\footnotetext{
*This work was in part supported by the Natural Sciences and Engineering Research Council of Canada (NSERC), the Mathematics of Information Technology and Complex Systems (MITACS), and the European Research Council StG 259559 and OTKA K109782.
} 


\begin{abstract}
Many vaccine preventable diseases require a booster dose in addition to the primary vaccination in order to provide adequate level of protection. However, the optimal timing of booster dose after primary vaccination is often difficult to determine. Here we propose an agent-based modelling framework to investigate the timing of booster vaccination, based on the efficacy of primary vaccine series and the coverage of booster dose. We show that these factors can significantly affect the long-term epidemological outcomes of vaccination. We represent our results by simulating the model for the dynamics of Heamophilus influenzae serotype b (Hib), using parameter estimates for natural history of the disease, its transmissibility, and the protection efficacy of vaccine. We show that, for estimated vaccine protection efficacy against Hib, if the primary and booster coverages are maintained at high levels, a longer time interval between primary and booster doses leads to better outcomes and potentially elimination of the disease. The length of this time interval is however dependent on the coverage of booster vaccination.
\end{abstract}

\title{
1. Introduction
}

Vaccination remains the most successful preventive measure against many infectious diseases in modern medicine ${ }^{1}$. Despite successful implementation of vaccination programs, elimination of some disease has proven challenging, with instances of resurgence occurring in different geographic locations ${ }^{2,3,4}$. Several factors have been investigated as potential explicators for persistence and recurrent outbreaks of vaccine-preventable diseases, including incomplete protection efficacy of primary series, inadequate coverage of booster doses, waning immunity over time, and the period of vaccineinduced protection that may be significantly shorter than the average lifetime of the population ${ }^{5,4}$. However, given these factors, the time interval between primary series and booster vaccination may significantly influence both short- and long-term disease outcomes.

In this study, we develop an agent-based modelling (ABM) framework to investigate how the interval between primary and booster vaccination affects disease dynamics. While importance of age at vaccination has been well documented, the optimal timing of booster doses remains unclear in several vaccine-preventable diseases ${ }^{6,7,4}$. For this study, we considered Haemophilus influenzae serotype b (Hib), for which primary and booster vaccination has been implemented in routine infant immunization programs in many countries. Yet, the elimination has not been achieved, notwithstanding substantial levels of routine primary vaccine series ${ }^{2,3,4}$. We included key biological and epidemiological determinants of Hib in the model, and parameterize it with the previously published estimates to investigate the effect of timing for booster dose after primary vaccination on 


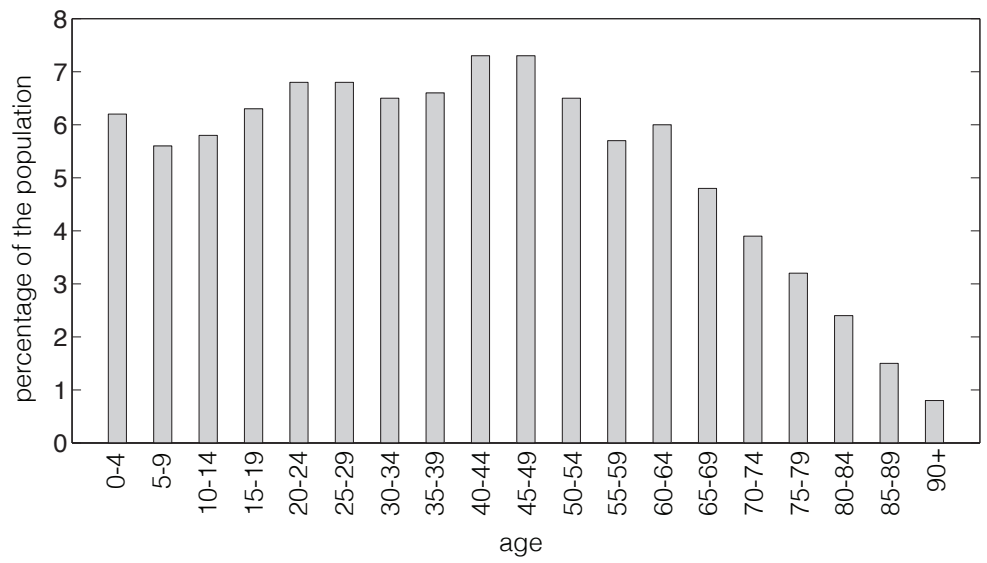

Figure 1. Age distribution of the United Kingdom population according to the 2011 census data: https://en.wikipedia.org/wiki/Demography_of_the_United_Kingdom.

the prevalence of disease. We illustrate our results using stochastic MonteCarlo simulations, and argue that the timing of booster dose may be an essential component of vaccination programs, whose optimal determination depends critically on the protection efficacy of primary vaccine series, and the coverage of booster vaccination.

\section{Methodology}

\subsection{Agents-environment framework}

The general framework of the model includes two main entities: (i) a simulated environment represented by a two dimensional lattice and (ii) the individuals that are located in the lattice. Each individual is characterized by their time-sensitive information vector, which encapsulates a plethora of information including the current health status, age, and the immune protection level. The size of the lattice was set to $317 \times 317$, resulting in an environment with 100489 individuals with age distributions similar to the UK population (Figure 1). The interaction between individuals was modelled through contacts with their immediate neighbours or random contacts on the lattice.

\subsection{Disease model}

In construction of the $\mathrm{ABM}$ framework, we consider the disease model with several epidemiological statuses of the individuals, based on which 


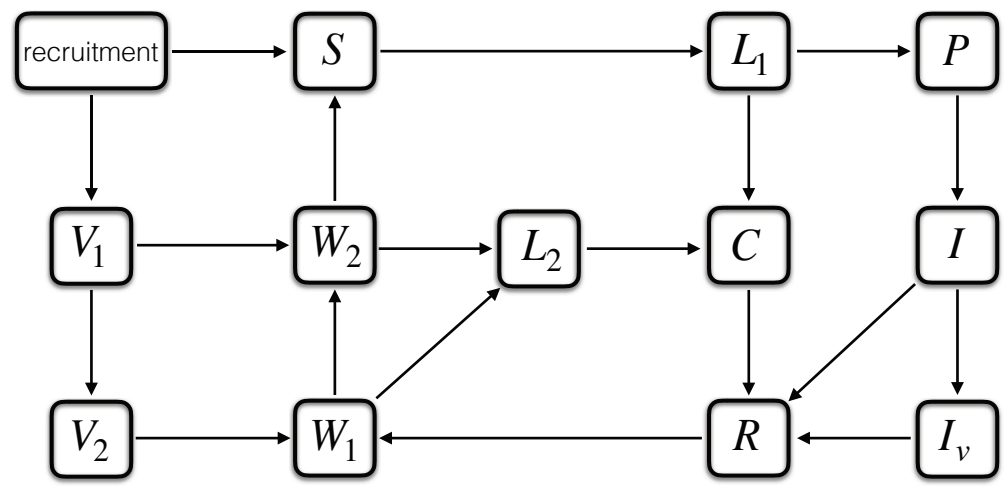

Figure 2. Schematic model diagram for transitions between epidemiological compartments in the disease model of the ABM framework.

the population is divided into several compartments. The disease model includes compartments of susceptible $(S)$, primary latent $\left(L_{1}\right)$, secondary latent $\left(L_{2}\right)$, pre-symptomatic $(P)$, asymptomatic referred to as carriage $(C)$, symptomatic $(I)$, invasive disease $\left(I_{v}\right)$, recovered $(R)$, vaccinated with primary series $\left(V_{1}\right)$, vaccinated with booster dose $\left(V_{2}\right)$, partially protected stage $1\left(W_{1}\right)$, and partially protected stage $2\left(W_{2}\right)$. Disease transmission occurs as a result of contact between infectious individuals (either in carriage of symptomatic stages), and those with no or incomplete protection (i.e., susceptible or partially protected individuals). The movements of individuals between the epidemiological compartments of the model are schematically represented in Figure 2.

Once exposed to the disease with successful transmission, the individuals will enter one of the latent stages (primary or secondary), depending on whether or not they have any protection level as a result of previous natural infection or vaccination. Individuals in the latent stages are assumed to be non-infectious and therefore cannot transmit the disease. Infected individuals with no pre-existing immune protection will proceed to pre-symptomatic infection and develop either symptomatic disease (with clinical manifestation) or remain asymptomatic (without clinical symptoms) for the entire duration of infectious period. If treatment is not sought, symptomatic individuals may develop invasive disease with potentially fatal outcomes. We assumed that infected individuals with pre-existing immune protection will develop only asymptomatic disease and remain carriage. Following recov- 
ery from infection, individuals will have a fixed period of full protection, during which no infection can occur. We also assumed this immune protection wanes over time and once the period of full protection has elapsed, the individuals will have a fixed period of partial protection, during which the infection can occur, albeit with lower transmission probability compared to fully susceptible individuals. This is followed by a second period of partial protection, sampled for each individual independently, with a lower protection level compared to the first period of partial protection. After the second protection period has elapsed, individuals will become susceptible to the disease again.

\subsection{Vaccination model}

For evaluating the effect of vaccination, we implemented primary vaccination for infants within their 6 months of birth ${ }^{8}$. Booster vaccination was offered only to those who had received primary vaccination. Vaccination of infants with primary series will lead to a fixed duration of partial protection, and followed by the second period of partial protection (as described above for natural infection) without booster vaccination. Those who received booster vaccination will be fully protected for a fixed length of time, and follow the same path of partial protection as described for individuals recovered from natural infection.

\subsection{Computational implementation}

The model was calibrated with the reproduction number $R_{0}=1.3^{9}$ to obtained the baseline transmissibility $\beta=7 \times 10^{-3}$ in the absence of any interventions (i.e., treatment or vaccination). Simulations were initialized with $C(0)=15$ for infection. The parameters governing the dynamics of the model were drawn from previously published literature, and are described in Table 1 . The model includes both disease induced and natural deaths. All deaths were replaced with newborns, thus maintaining a constant population size. The probability of natural death was calculated from its associated distribution with the median life expectancy of 60 years.

The success of transmission was determined using rejection samplingbased (Bernoulli) trials where the chance of success is defined by a transmission probability distribution. In the absence of pre-existing immune protection, the spread of disease between a susceptible-infectious pair of individuals was calculated using the baseline transmission probability

$$
P_{0}=1-\left(1-\eta_{p} \beta\right)^{K_{p}}\left(1-\eta_{c} \beta\right)^{K_{c}}(1-\beta)^{K_{s}}
$$


where $K_{p}$ is the number of contacts with pre-symptomatic individuals, $K_{c}$ is the number of contacts with individuals in the stage of carriage, $K_{s}$ is the number of contacts with symptomatic individuals, and $\eta_{p}$ and $\eta_{c}$ are respectively the relative reduction of infectiousness in pre-symptomatic and carriage individuals compared with symptomatic individuals. However, the probability $P_{0}$ is subject to the immune protection level of the individuals. To account for the effect of immunity in the reduction of disease transmissibility, we calculated the transmission probability by

$$
P_{V_{1}}=\left(1-\eta_{v_{1}}\right) P_{0}
$$

for primary vaccinated individuals, and by

$$
\begin{aligned}
& P_{W_{1}}=\left(1-\eta_{w_{1}}\right) P_{0}, \\
& P_{W_{2}}=\left(1-\eta_{w_{2}}\right) P_{0},
\end{aligned}
$$

for individuals in the first and second stages of partial protection, where $\eta_{v_{1}}, \eta_{w_{1}}$, and $\eta_{w_{2}}$ are respectively the relative reductions of susceptibility to infection (as a results of immune protection) compared with fully susceptible individuals.

\subsection{Parmeterization and timing of booster vaccination}

We parameterize the model with estimates for various epidemiological stage and duration of protection induced by vaccination or natural infection. These parameters and their sources are provided in Table 1. A number of parameters were varied in our simulations to evaluate the effect of the primary vaccine series and the subsequent booster dose. The timing for booster dose was varied between 6 to 48 months after primary vaccination. We assumed three different profiles of booster dose coverage (Figure 3): (i) a fixed coverage the same as the primary vaccine coverage regardless of the time for booster dose (Figure 3, dark grey bars); (ii) a reduced coverage of booster that follows an exponential declining trend (Figure 3, black bars); and (iii) a reduced coverage of booster that follows a declining trend similar to the inverse of the logistic function (Figure 3, light grey bars). The reduction in booster dose coverage may reflect a number of factors, including higher rates of refusal to receive additional vaccine doses following primary series, which may be affected by delay in booster schedule. 
Table 1. Description of model parameters and their associated values (ranges) extracted from the published literature.

\begin{tabular}{llc} 
parameter description & value (range) & source \\
\hline basic reproduction number & $1.3(1.1-1.5)$ & 10,11 \\
\hline coverage of primary vaccination & $0.95(0-1)$ & - \\
\hline $\begin{array}{l}\text { coverage of booster vaccination } \\
\text { latent period }\end{array}$ & $2(1-3)$ day & - \\
$\begin{array}{l}\text { pre-symptomatic period } \\
\text { infectious period of carriage and }\end{array}$ & $1.5(1-2)$ days & 12 \\
$\begin{array}{l}\text { symptomatic infection } \\
\text { age at completion of primary vaccine series }\end{array}$ & $14-70$ days & $12,13,2$ \\
$\begin{array}{l}\text { timing of booster coverage post } \\
\text { primary vaccination }\end{array}$ & variable $(0-48)$ months & - \\
$\begin{array}{l}\text { fixed period of full protection following } \\
\text { recovery from infection }\end{array}$ & 2 years & 12,2 \\
$\begin{array}{l}\text { fixed period of full protection following } \\
\text { booster vaccination }\end{array}$ & 6 years & $12,2,9,13$ \\
$\begin{array}{l}\text { fixed period of partial protection stage } 1 \\
\text { period of partial protection stage } 2\end{array}$ & 2 years & 12 \\
$\begin{array}{l}\text { level of immune protection following } \\
\text { primary vaccination }\end{array}$ & $2-8$ years & 12 \\
$\begin{array}{l}\text { level of immune protection during } \\
\text { partial protection stage } 1\end{array}$ & $0.8(0.6-0.9)$ & $12,2,9,13$ \\
$\begin{array}{l}\text { level of immune protection during } \\
\text { partial protection stage } 2\end{array}$ & $0.6(0.3-0.9)$ & 12,2 \\
$\begin{array}{l}\text { infectious period following start of treatment } \\
\text { in symptomatic infections }\end{array}$ & 2 days & 12,2 \\
$\begin{array}{l}\text { probability of developing carriage without } \\
\text { pre-existing immune protection }\end{array}$ & $0.6-0.9$ & $?, 12$ \\
\hline & $0.3(0.1-0.5)$ & 12,13 \\
\hline
\end{tabular}

\section{Results}

For each scenario of booster dose coverage (Figure 3), we ran stochastic simulations to obtain the average of 100 independent realizations. The model with vaccination was seeded with the initial conditions given by the components of the endemic equilibrium of the model without vaccination. For the scenarios simulated here, the timing for booster dose after primary vaccination was varied. Figure 4 shows the prevalence of carriage for 28 years following the start of vaccination, when the booster dose was offered with delay of 9 months, 24 months, or 36 months after primary vaccination. For all scenarios, the introduction of vaccination significantly reduced the prevalence of carriage in the absence of vaccination. 


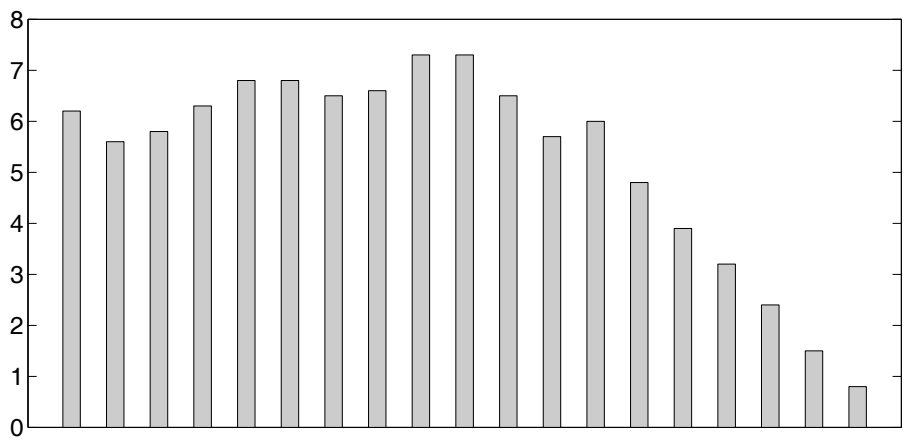

Figure 3. Time-dependent coverage of booster vaccination post primary vaccination.

When the booster dose coverage is maintained at 95\% (Figure 4A), we observed minimal differences with increasing delay in booster vaccination, with slight advantage in booster vaccination with longer delay (36 months). However, for a booster coverage that declines exponentially with delay (Figure 4B), we observed that early booster (9 months) outperforms other strategies with longer delays, contrasting the scenario of fixed booster coverage. As delay in timing of booster dose increases, a lower fraction of primary vaccinated individuals receives booster dose which leads to a higher prevalence of infection over time. For the scenario in which the coverage of booster dose reduces over time similar to the inverse of the logistic function (Figure 3 ), both strategies of early ( 9 months) and late (36 months) booster vaccination were outperformed by the strategy with an intermediate delay of 24 months. These results suggest that timing of booster vaccination could have a significant impact on the long-term disease outcomes, and the optimal timing depends not only on the protection efficacy of the vaccine, but also on the coverage of the booster dose.

\section{Concluding Remarks}

There is evidence accumulating that the booster vaccine schedule requires evaluation for several vaccine-preventable diseases to improve the effect of vaccination campaigns ${ }^{6,7,4}$. Previous studies have shown the time interval between the primary vaccination and booster dose could play a vital role in the long-term dynamics of $\mathrm{Hib}^{14}$, and a large delay in booster vaccination may result in a longer protection period during lifetime ${ }^{15}$. However, a larger delay may lead to a lower fraction of primaries receiving the booster 
dose as a results of parental hesitancy and refusal with subsequent increase in the number of unvaccinated and undervaccinated individuals ${ }^{16,17,18}$.

In this study, we developed an agent-based model of disease transmission to investigate the vaccination and timing of booster dose on the prevalence of long-term infection dynamics. Considering several biological and epidemiological aspects of natural and vaccine-induced immunity, we simulated the model for various delays in booster dose vaccination using parameter values estimated for Heamophilus influenza serotype b published in the literature.

Simulation results indicate that the timing of booster dose can significantly influence the prevalence of infection in the population. While we compared the outcomes of different strategies for booster schedule, determination of the optimal timing for booster vaccination remains a challenging task. Although we have shown in a number of scenarios simulated here that the delay in booster vaccination may be beneficial in reducing the disease prevalence, the coverage of booster remains a key determining factor that may be influenced by the delay in booster vaccination ${ }^{16,17,18}$. If the coverage of booster vaccination is expected to drop significantly over time, our simulations illustrate that early booster schedule may be essential to achieve the greatest reduction of the disease prevalence.

The objective of this study was not to determine the optimal timing of a booster dose vaccination, but rather illustrate the complexity of the disease dynamics in the context of vaccination and highlight the challenge in optimizing immunization programs with long-lasting effects in prevention and disease elimination.

The model proposed here could be further evaluated with variability is parameter space to better understand the sensitivity of outcomes with respect to the infection and vaccination specific parameters. This understanding could help to more accurately quantify the benefit of different vaccination programs and booster schedules. 
(A)

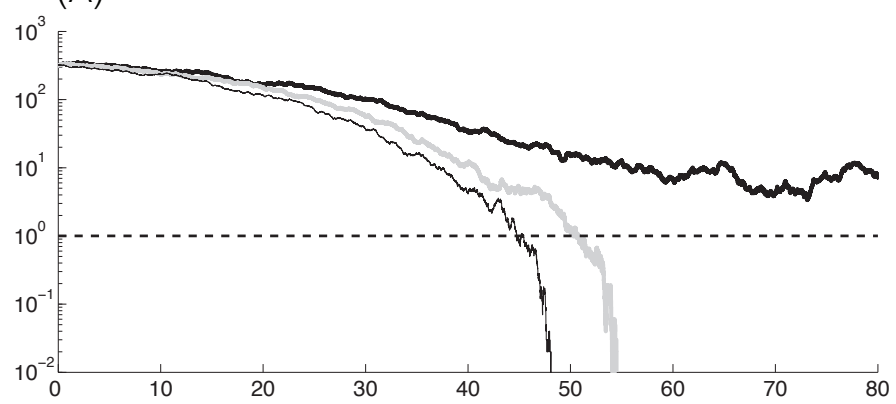

(B)

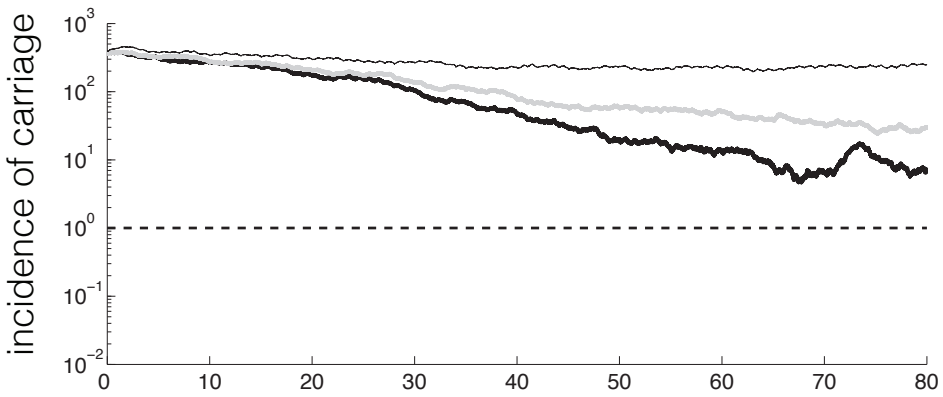

(C)

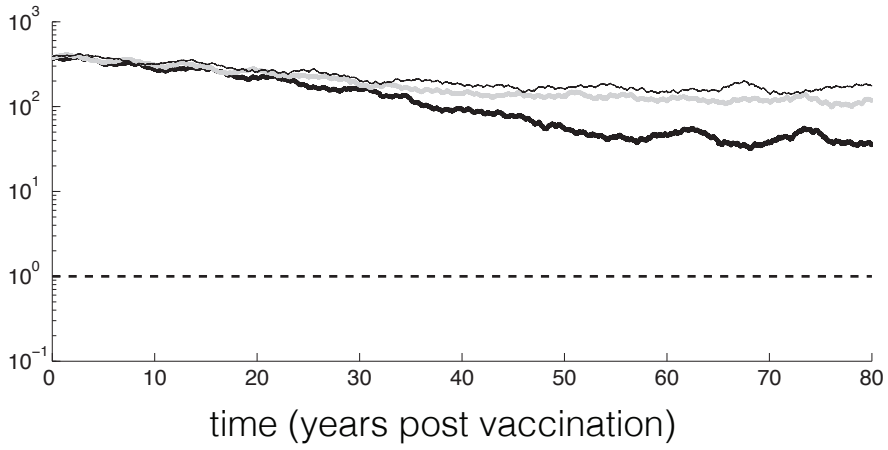

Figure 4. Incidence of carriage with three different booster vaccination coverage scenarios: (A) Fixed (95\%) coverage; (B) declining coverage similar to the inverse of logistic function; and (C) exponentially declining coverage (as illustrated in Figure 3). Delay in booster dose is 9 months (bold black curves), 24 months (gray curves), and 36 months (black curve). 


\section{References}

1. J. Ehreth, The global value of vaccination. Vaccine, 21 (7): 596-600, 2003.

2. M. L. Jackson et al., Modeling insights into haemophilus influenzae type b disease, transmission, and vaccine programs. Emerging Infectious Disease, 18 (1): 13-20, 2012.

3. M. A. Riolo et al., Can vaccine legacy explain the british pertussis resurgence? Vaccine, 31 (49): 5903-5908, 2013.

4. M. A. Riolo, P. Rohani, Combating pertussis resurgence: One booster vaccination schedule does not fit all. Proceedings of the National Academy of Sciences, 112 (5): E472-E477, 2015.

5. M. E. Alexander et al., Modelling the effect of a booster vaccination on disease epidemiology. Journal of Mathematical Biology, 52 (3): 290-306, 2006.

6. N. Low et al., Comparing haemophilus influenzae type b conjugate vaccine schedules: a systematic review and meta-analysis of vaccine trials. The Pediatric Infectious Disease Journal, 32 (11): 1245-1256, 2013.

7. C. Jackson et al., Effectiveness of haemophilus influenzae type b vaccines administered according to various schedules: systematic review and metaanalysis of observational data. The Pediatric Infectious Disease Journal, 32 (11): 1261-1269, 2013.

8. World Health Organization et al., Who recommendations for routine immunization-summary tables. Geneva: WHO, 2016.

9. A. Konini et al., Dynamics of naturally acquired antibody against haemophilus influenzae type a capsular polysaccharide in a canadian aboriginal population. Preventive Medicine Reports, 3: 145-150, 2016.

10. C. P. Farrington et al., Estimation of the basic reproduction number for infectious diseases from age-stratified serological survey data. Journal of the Royal Statistical Society: Series C (Applied Statistics), 50 (3): 251-292, 2001.

11. D. S. Stephens, Protecting the herd: the remarkable effectiveness of the bacterial meningitis polysaccharide-protein conjugate vaccines in altering transmission dynamics. Transactions of the American Clinical and Climatological Association, 122: 115, 2011.

12. A. Konini, S. M. Moghadas, Modelling the impact of vaccination on curtailing haemophilus influenzae serotype 'a'. Journal of Theoretical Biology, 387: 101$110,2015$.

13. T. Leino et al., Dynamics of natural immunity caused by subclinical infections, case study on haemophilus influenzae type b (hib). Epidemiology and Infection, 125 (03): 583-591, 2000.

14. S. P. Fitzwater et al., Haemophilus influenzae type b conjugate vaccines: considerations for vaccination schedules and implications for developing countries. Human Vaccines, 6 (10): 810-818, 2010.

15. N. Charania, S. M. Moghadas, Modelling the effects of booster dose vaccination schedules and recommendations for public health immunization programs: the case of haemophilus influenzae serotype b. BMC Public Health, in review, 2016.

16. S. B. Omer et al., Vaccine refusal, mandatory immunization, and the risks 
of vaccine-preventable diseases. New England Journal of Medicine, 360 (19): 1981-1988, 2009.

17. E. Dubé et al., Vaccine hesitancy: an overview. Human Vaccines \& Immunotherapeutics, 9 (8): 1763-1773, 2013.

18. E. C. Briere et al., Prevention and control of haemophilus influenzae type b disease: recommendations of the advisory committee on immunization practices (acip). MMWR Recommendations \& Reports, 63 (RR-01): 1-14, 2014. 